\title{
16. \\ Sur le théorême de Wilson.
}

(Par Mr. Brennecke, professeur de math. à Berlin.)

Le théorême de Wilson a été généralisé par Mr. Gau/s comme il suit: "Le produit de tous les nombres premiers à un nombre donné „ $N$ et moindres que ce nombre est congru suivant $N$ à l'unité prise „positivement ou négativement. L'unité doit être prise négativement, " quand $N$ est de la forme $p^{\alpha}$ ou $2 p^{\alpha}, p$ étant un nombre premier „impair, ou encore quand $N=4$; dans tous les autres cas l'unité „ doit être prise positivement."

\section{Notions préliminaires.}

§. 1. La congruence $x^{2} \equiv 1\left(\bmod . p^{\alpha}\right), p$ étant un nombre premier impair, n'a que ces deux racines $x=1$ et $x=p^{\alpha}-1$.

La différence des deux diviseurs $x+1$ et $x-1$ de $x^{2}-1$ étant égale à 2 , ils ne peuvent être à la fois des multiples de $p, p$ étant un nombre premier impair, $x$ étant moindre que $p^{\alpha}, x+1$ ne devient divisible par $p^{\alpha}$ que pour $x=p^{\alpha}-1$, et $x-1$ ne devient divisible par $p^{\alpha}$ que pour $x=1$.

§. 2. La congruence $x^{2} \equiv 1\left(\bmod .2 p^{\alpha}\right), p$ étant un nombre premier impair, n'a que ces deux racines $x=1$ et $x=2 p^{\alpha}-1$.

La différence des deux diviseurs $x+1$ et $x-1$ de $x^{2}-1$ étant égale à $2, p$ étant un nombre premier impair, ces deux diviseurs ne peuvent être à la fois des multiples de $p . x+1$ ne devient divisible par $2 p^{\alpha}$ que pour $x=2 p^{\alpha}-1, x-1$ ne devient divisible par $2 p^{\alpha}$ que pour $x=1$. $x+1$ et $x-1$ étant à la fois des nombres pairs ou impairs, $p^{a}$ étant toujours un nombre impair, l'un de ces diviseurs ne peut être divisible par 2 tandis que l'autre est égal à $p^{\alpha}$.

§. 3. La congruence $x^{2} \equiv 1\left(\bmod .2^{\alpha}\right)$ a toujours pour $a>2$ quatre racines: $1,2^{\alpha-1}-1,2^{\alpha-1}+1,2^{\alpha}-1$, mais il n'en existe jamais d'autres. La congruence $x^{2} \equiv 1$ (mod. 4) n'a que ces deux racines $x=1, x=3$.

En effet toute racine de $(x+1)(x-1) \equiv 0\left(\bmod .2^{\alpha}\right)$ doit être un nombre impair c'est-à-dire de la forme $2 k+1, k$ étant un nombre en- 
tier quelconque ou zéro, On aura dono $(2 k+2) 2 k \equiv 0\left(\bmod .2^{c}\right)$, ou pour $\alpha>2, k(k+1) \equiv 0\left(\bmod .2^{\alpha-2}\right)$. Pour $k=0$, on a $x=1$, pour $k=2^{\alpha-2}-1$, on a $x=2^{\alpha-1}-1$, mais il est évident que $k>0$ et et $k<2^{\alpha-2}-1$ ne satisfait pas à la congruence $k(k+1) \equiv 0\left(\bmod .2^{\alpha-2}\right)$, puisqu'il n'y a qu'un seul des diviseurs $k$ et $k+1$ qui puisse être divisible par 2. Pour $k=2^{\alpha-2}$ on a $x=2^{\alpha-1}+1$, pour $k=2^{\alpha-1}-1$ on a $x=2^{\alpha}-1$, mais il est évident que $k>2^{\alpha-2}$ et $k<2^{\alpha-1}-1$ ne satisfait pas à la congruence $k(k+1) \equiv 0\left(\bmod .2^{\alpha-2}\right)$.

\$. 4. Si deux nombres $a$ et $b$ sont congrus suivant le module $M$, et que l'un soit un nombre premier à $M$, l'autre doit être aussi un nombre premier avec $M$. Si au contraire l'un a un diviseur commun avec $M$, l'autre dait avoir le mệe diviseur commun avec $M$, P. c. puisque tout terme de la suite

$$
\text { 1. } a, a+1, a+2, a+3, \ldots a a+(M-1)
$$

est congru à un terme de la suite selon le module $M$ (mais seulement à un)

$$
\text { 2. } 1,2,3, \ldots M
$$

et que cette propriété est réciproque entre les deux suites (1.) et (2.), il $y$ aura dans chacune un nombre égal de nombres premiers avec $M$, c'est-à-dire (si $\left.M=q^{\beta} v^{\gamma} \ldots ..\right) q^{\beta-1} v^{\gamma-1} \ldots .(q-1)(v-1) \ldots(.$, que a soit un nombre entier positif ou négatif quelconque.

On sait que si $m$ est un nombre premier avec $M$, les termes de la suite

$$
\text { 3. } \quad 0 . m, 1 . m, 2 . m, \ldots(M-1) . m
$$

donnent d'après le module $M$ pour restes tous les nombres de la suite (2) en substituant $M$ à la place de zéro, et que ohaque reste ne s'y trouve qu'une seule fois. Tout terme de la suite (3,) est donc congru selon le module $M$ ì un terme mais seulement ì un terme de la suite (2.). Cette propriété est réciproque entre les suites (2.) et (3.).

Il en résulte que tout terme de la suite

$$
\text { 4. } a+0 . m, a+1 . m, a+2 . m, \ldots, a+(M-1) \cdot m
$$

sera congru à un terme de la suite (1.) selon le module $M$ et réciproque. ment, n'importe la valeur de $a$, pourvu que a soit un nombre entier. Il s'ensuit que la suite (4.) renferme autant de nombres premiers avec $\boldsymbol{M}$ que la suite (2.) c'est-ä-dire $q^{p-1} v \gamma^{-1} \ldots . .(q-1)(p-1) \ldots .$.

6. 5. En supposant

$$
\text { 1. } \quad \mathrm{N}=p^{a} \cdot M \text { of } M=q^{\beta} v^{\gamma} \ldots \text {, }
$$


$p, q, \vartheta, \ldots$ étant des nombres premiers différents, $a, \beta, \gamma, \ldots$ des nombres entiers, et tous les nombres premiers avec $p^{\alpha}$ moindres que $p^{\alpha}$ représentés par

$$
\text { 2. } r_{1}, r_{2}, r_{3}, \ldots . r_{\mu}\left(\mu=p^{\alpha-1}(p-1)\right) \text {, }
$$

il sera d'après le $\S .4$. facile de construire un tableau qui renferme tous les nombres premiers avec $\boldsymbol{p}^{\alpha}$ moindres que $\boldsymbol{N}$ savoir:

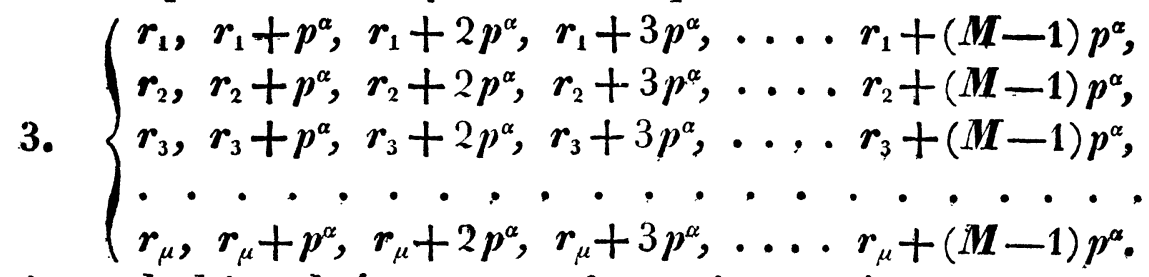

Car si $a=k . d+c, k$ étant un nombre entier ou zéro, $a$ sera un nombre premier avec $d$, si $c$ l'est avec $d$, si au contraire $c$ et $d$ ont le plus grand diviseur commun $\delta$, $a$ et $d$ auront aussi le plus grand diviseur commun $\delta$. Le tableau précédent en contenant tous les nombres premiers avec $\boldsymbol{p}^{\alpha}$ moindres que $N$ renfermera tous les nombres premiers avec $N$ moindres que $N$, mais contiendra aussi beaucoup de nombres qui ne sont pas des nombres premiers avec $N$.

§. 6. D'après ce que nous avons prouvé (\$. 4.) en mettant $m=p^{\alpha}$, $p^{\alpha}$ étant un nombre premier avec $q^{\beta} v^{\gamma}$... chaque colonne horizontale du tableau de $\S .5$. renferme $q^{\beta-1} v^{\gamma-1} \ldots .(q-1)(v-1) \ldots .$. nombres premiers avec $N$, il y a $p^{\alpha-1}(p-1)$ colonnes horizontales, donc le tableau de $\S .5$. renferme $p^{\alpha-1} q^{\beta-1} v^{\gamma-1} \ldots(p-1)(q-1)(v-1) \ldots$. nombres premiers différents avec $N$ moindres que $N$. Il en résulte que ce tạbleau contient tous les nombres premiers avec $N$ moindres que $N$,

\section{Démonstration du théorême énoncé.}

§. 7. Soit $p$ un nombre premier impair, et soient

$$
\text { 1. } r_{1}, r_{2}, r_{3}, \ldots . r_{\mu}
$$

tous les nombres premiers avec $p^{\alpha}$ moindres que $p^{\alpha}$, dont $r_{1}=1, r_{\mu}=p^{\alpha}-1$, $\mu_{1}=p^{\alpha-1}(p-1)$ sera un nombre pair. Nous allons à présent considérer les nombres

$$
\text { 2. } r_{2}, r_{3}, r_{4}, \ldots . r_{\mu-1} \text {, }
$$

dont le nombre sera égalenent pair. Soit $s$ un des nombres $2,3,4, \ldots$ $\ldots(\mu-1)$, la congruence

$$
\text { 3. } \quad r_{s} x \equiv 1 \quad\left(\bmod , p^{\alpha}\right)
$$

Crelle's Journal d. M. Ba. XIX. Hift. 4. 
ne sera satisfaite que dans le cas où $x$ est un nombre premier avec $p^{\alpha}$. $x$ ne pourra être égal ni à 1 ni à $p^{\alpha}-1$, car dans le premier cas, $\boldsymbol{r}_{s}$ serait égal à 1 , dans le second, $\boldsymbol{r}_{s}$ serait égal ì $p^{\alpha}-1 . x$ ne pourra être égal à $r_{s}$, puisque d'après $\S$. 1. la congruence $x^{2} \equiv 1\left(\bmod . p^{\alpha}\right)$ n'est satisfaite que par $x=1$ et $x=p^{\alpha}-1$. La congruence (3.) étant du premier dégré et $r_{s}$ étant un nombre premier avec $p^{\alpha}$, n'admet qu'une seule racine. P. c. $r_{s}$ et $x$ étant tous les deux des nombres différents contenus en (2.), on pourra former $\frac{\mu-2}{2}$ congruences de la forme (3.) qui ne contiendront que toutes les valeurs de (2.), mais dans lesquelles chaque valeur ne se trouve qu'une seule fois. Le produit de ces $\frac{\mu-2}{2}$ congruences donne

$$
\text { 4. } r_{2} \cdot r_{3} \cdot r_{4} \ldots r_{\mu-1} \equiv 1\left(\bmod \cdot p^{a}\right) \text {, }
$$

laquelle multipliée par la congruence $1 \cdot\left(p^{\alpha}-1\right) \equiv-1\left(\bmod . p^{\alpha}\right)$ ou par $r_{1} \cdot r_{\mu} \equiv-1\left(\bmod \cdot p^{\alpha}\right)$ donne

$$
\text { 5. } r_{1} . r_{2} . r_{3} \ldots r_{\mu} \equiv-1\left(\bmod . p^{\alpha}\right), \quad \mu^{\alpha-1}=p^{\alpha-1}(p-1) \text {. }
$$

En appliquant le même raisonnement au module $2 p^{\alpha}, p$ étant un nombre premier impair, puisque la congruence $x^{z} \equiv 1\left(\bmod .2 p^{\alpha}\right)$ n'a que les racines 1 et $2 p^{\alpha}-1$ ( cf. §. 2.), on trouvera en désignant par (1.) tous les nombres premiers avec $2 p^{\alpha}$ moindres que $2 p^{\alpha}$,

$$
\text { 6. } r_{1} \cdot r_{2} \cdot r_{3} \ldots r_{\mu}=-1\left(\bmod .2 p^{\alpha}\right), \quad \mu=\left(p^{\alpha-1}\right)(p-1) \text {. }
$$

La même proposition a encore lieu pour le module 4 puisque $1.3 \equiv-1$ $(\bmod , 4)$.

Le même raisonnement appliqué au module $2^{\alpha}(\alpha>2)$ nous donnera, puisque la congruence $x^{2} \equiv 1\left(\bmod .2^{\alpha}\right)$, a étant plus grand que 2, n'a que les quatre racines $1,2^{\alpha-1}-1,2^{\alpha-1}+1,2^{\alpha}-1$, et que

$$
\text { 7. } 1 .\left(2^{\alpha}-1\right) \equiv-1\left(\bmod .2^{\alpha}\right) \text { et }\left(2^{\alpha-1}-1\right)\left(2^{\alpha-1}+1\right) \equiv-1\left(\bmod .2^{\alpha}\right) \text {, }
$$
en désignant par (1.) tous les nombres premiers avec $2^{\alpha}$ moindres que $2^{\alpha}$, 8. $r_{1} \cdot r_{2} \cdot r_{3} \ldots r_{\mu} \equiv 1\left(\bmod .2^{\alpha}\right), \alpha>2, \mu=2^{\alpha-1}$.

8. 8. Après avoir considéré les cas de $N=p^{\alpha} ; N=2 p^{\alpha}$ ( $p$ étant dans ces deux cas un nombre premier impair), $N=4, N=2^{\alpha}(\alpha>2)$, nous allons considérer tous les autres cas possibles compris sous la forme de $N=p^{\alpha} q^{\beta} v^{\gamma} \ldots, p, q, v, \ldots$ étant des nombres premiers différents. Dans tous ces derniers cas $q^{\beta-1} v^{\gamma-1} \ldots(q-1)(v-1) \ldots$ est un nombre pair, c'est-à-dire dans chaque colonne horizontale du tableau de $\S .5$. il y a un nombre pair de nombres premiers avec $N$ moindres que $N$. 
Tous les nombres de la première colonne horizontale du tableau de $\S .5$. sont congrus avec $r_{1}$ suivant le module $p^{\alpha}$, tous ceux de la seconde colonne avec $r_{2}$, ceux de la troisième avec $r_{3}$, etc. En ne prenant dans toutes les colonnes horizontales que les nombres premiers arec $\boldsymbol{N}$, chaque colonne donne $q^{\beta-1} v^{\gamma-1} \ldots(q-1)(v-1)$ etc. congruences selon $p^{\alpha}$. Le produit de toutes ces congruences donne, en désignant par $[N]$ le produit de tous les nombres premiers avec $N$ moindres que $N$,

1. $[\mathrm{N}] \equiv\left(r_{1}, r_{2}, r_{3} \ldots . r_{\mu}\right)^{q^{\beta-1} v^{\gamma-1} \ldots(q-1)(v-1) \ldots .} \quad\left(\bmod . p^{\alpha}\right)$, lequel d'après $\S .7 .$, puisque $q^{\beta-1} v^{\gamma-1} \ldots .(q-1)(v-1) \ldots$ est un nombre pair, donne

$$
\text { 2. }[\mathbf{N}] \equiv 1\left(\bmod \cdot p^{\alpha}\right) \text {. }
$$

On troure par des raisonnements analogues

$$
\text { 3. }\left\{\begin{array}{cc}
{[N] \equiv 1} & \left(\bmod p^{\alpha}\right), \\
{[N] \equiv 1 \quad\left(\bmod q^{\beta}\right),} \\
{[N] \equiv 1 \quad\left(\bmod v^{\gamma}\right),} \\
\ldots \ldots \ldots
\end{array}\right.
$$

Les modules de toutes ces congruences étant entre eux des nombres premiers, il en résulte

$$
\text { 4. }[N] \equiv 1\left(\bmod . p^{\alpha} q^{\beta} v^{\gamma} \ldots \text { ou } \bmod . N\right) \text {, }
$$

ce qu'il s'agissait de prouver. 